\title{
Constraints on the Adiabatic Temperature Change in Magnetocaloric Materials
}

\author{
Nielsen, Kaspar Kirstein; Bahl, Christian Robert Haffenden; Smith, Anders
}

Published in:

Physical Review B Condensed Matter

Link to article, DOI:

10.1103/PhysRevB.81.054423

Publication date:

2010

Document Version

Publisher's PDF, also known as Version of record

Link back to DTU Orbit

Citation (APA):

Nielsen, K. K., Bahl, C. R. H., \& Smith, A. (2010). Constraints on the Adiabatic Temperature Change in Magnetocaloric Materials. Physical Review B Condensed Matter, 81(5), 054423.

https://doi.org/10.1103/PhysRevB.81.054423

\section{General rights}

Copyright and moral rights for the publications made accessible in the public portal are retained by the authors and/or other copyright owners and it is a condition of accessing publications that users recognise and abide by the legal requirements associated with these rights.

- Users may download and print one copy of any publication from the public portal for the purpose of private study or research.

- You may not further distribute the material or use it for any profit-making activity or commercial gain

- You may freely distribute the URL identifying the publication in the public portal 


\title{
Constraints on the adiabatic temperature change in magnetocaloric materials
}

\author{
K. K. Nielsen* \\ Department of Mechanical Engineering, Technical University of Denmark, Building 425, Niels Koppels Alle, \\ DK-2800 Kongens Lyngby, Denmark \\ C. R. H. Bahl and A. Smith \\ Fuel Cells and Solid State Chemistry Division, Ris $\phi$ National Laboratory for Sustainable Energy, Technical University of Denmark, \\ Frederiksborgvej 399, DK-4000 Roskilde, Denmark
}

(Received 8 July 2009; revised manuscript received 14 December 2009; published 17 February 2010)

\begin{abstract}
The thermodynamics of the magnetocaloric effect implies constraints on the allowed variation in the adiabatic temperature change for a magnetocaloric material. An inequality for the derivative of the adiabatic temperature change with respect to temperature is derived for both first- and second-order materials. For materials with a continuous adiabatic temperature change as a function of temperature, this inequality is shown to hold for all temperatures. However, discontinuous materials may violate the inequality. We compare our results with measured results in the literature and discuss the implications of the result. Similar inequalities hold for barocaloric and electrocaloric materials.
\end{abstract}

DOI: 10.1103/PhysRevB.81.054423

PACS number(s): 75.30.Sg, 77.70.+a

\section{INTRODUCTION}

The magnetocaloric effect is due to the coupling between the magnetic and the lattice degrees of freedom in a solid. That this coupling can induce a field-dependent temperature change in a magnetic sample placed in an external magnetic field may be heuristically understood by an entropy argument. ${ }^{1}$ The total entropy of a magnetic solid can often to a good approximation be divided into contributions arising from the lattice degrees of freedom (i.e., from the phonons) and the magnetic degrees of freedom (the spin system). If there are extended electron states occupied, the entropy contribution of these must also be added. This division of the entropy is the basis for the following argument: when a magnetic material is placed in an external field $H$, the magnetic moments tend to align with the external field, thus decreasing the entropy associated with the magnetic degrees of freedom. Under adiabatic conditions the total entropy is constant, and the decrease in the magnetic part of entropy must be accompanied by an increase in the part of the entropy associated with the lattice degrees of freedom (as long as the electronic entropy can be assumed unchanged). This increase can be observed as a temperature increase in the material. However, it should be noted that in special cases, e.g., where competing phase transitions interact or if one of the phases is ferrimagnetic or antiferromagnetic, the temperature may actually decrease. ${ }^{2,3}$ One way for this to happen is when the change in entropy is due to the entropy difference between different electronic structures in phases separated by a firstorder phase transition. ${ }^{4}$

In this way, a magnetocaloric material is characterized thermodynamically by two fundamental materials properties, the isothermal entropy change when a field $H$ is applied, $\Delta S(T, H)$ (which is usually negative), and the adiabatic temperature change upon magnetization, $\Delta T_{\text {mag }}(T, H)$ (which is usually positive). We note in passing that if the starting field is not zero but $H_{0}$, the corresponding entropy and temperature change is $\Delta S\left(T ; H, H_{0}\right)=\Delta S(T, H)-\Delta S\left(T, H_{0}\right)$ and $\Delta T_{\text {mag }}\left(T ; H, H_{0}\right)=\Delta T_{\text {mag }}(T, H)-\Delta T_{\text {mag }}\left(T, H_{0}\right)$. These quanti- ties will be a function of both $H$ and $H_{0}$ and not just of their difference $\mathrm{H}-\mathrm{H}_{0}{ }^{5}$

It is the purpose of this paper to point out that the thermodynamics of the magnetocaloric effect constrains the possible variation in $\Delta T_{\text {mag }}$ with temperature. Since the argument is independent of the microscopic origin of the temperature change, the analysis is equally relevant for barocaloric or electrocaloric materials (a change in temperature with external pressure or electric field, respectively).

We start out by investigating the consequences of reversibility. Then we consider materials with first-order phase transitions which may possibly have an irreversible magnetocaloric effect, and discuss to what extent the findings are applicable to real materials. We compare our results with selected experimental results from the literature and finally discuss the implications of our analysis.

\section{CONSEQUENCES OF REVERSIBILITY}

Magnetocaloric materials exhibiting second-order (continuous) phase transitions have a reversible magnetocaloric effect. This amounts to the following: start out with a sample in a state with zero external field at a temperature $T_{0}$. When the sample is magnetized adiabatically, the temperature increases to $T^{*}=T_{0}+\Delta T_{\mathrm{mag}}\left(T_{0}, H\right)$. Now, upon adiabatic demagnetization the temperature drops to $T^{* *}=T^{*}+\Delta T_{\text {demag }}\left(T^{*}, H\right)$. If the process is reversible we must end in the state we started from, i.e., $T_{0}=T^{* *}$ or

$$
T_{0}+\Delta T_{\text {mag }}\left(T_{0}, H\right)+\Delta T_{\text {demag }}\left[T_{0}+\Delta T_{\text {mag }}\left(T_{0}, H\right), H\right]=T_{0},
$$

that is

$$
\Delta T_{\mathrm{mag}}\left(T_{0}, H\right)=-\Delta T_{\mathrm{demag}}\left[T_{0}+\Delta T_{\mathrm{mag}}\left(T_{0}, H\right), H\right] .
$$

Note that $\Delta T_{\text {demag }}$ is negative (if $\Delta T_{\text {mag }}$ is positive).

In the following, all $H$ dependence will be suppressed, and to emphasize the fact that $\Delta T_{\text {mag }}$ and $\Delta T_{\text {demag }}$ are differ- 

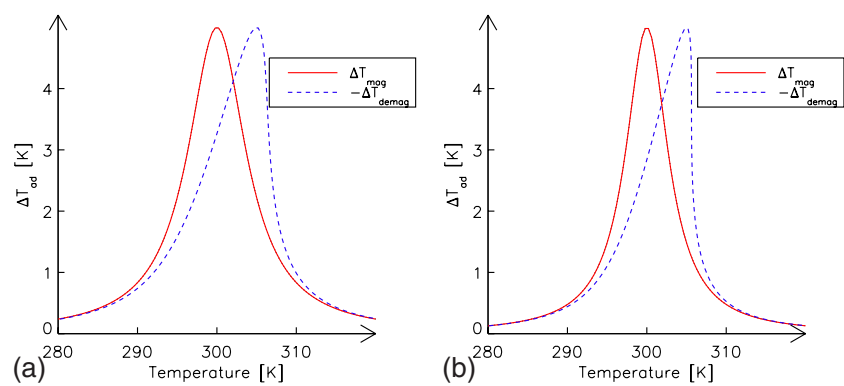

FIG. 1. (Color online) The adiabatic temperature change in a model magnetocaloric material both when magnetizing (full red line) and demagnetizing (dashed blue). (a) The slope of the $\Delta T_{\text {mag }}$ is greater than -1 for all temperatures. (b) The slope of the $\Delta T_{\text {mag }}$ curve is exactly -1 at a single temperature above $T_{*}$. This results in a demagnetization curve with a vertical tangent at the corresponding temperature.

ent functions of temperature the following notation will be introduced:

$$
\begin{gathered}
f(T) \equiv \Delta T_{\mathrm{mag}}(T, H), \\
g(T) \equiv \Delta T_{\mathrm{demag}}(T, H) .
\end{gathered}
$$

In terms of $f$ and $g$, the condition of reversibility becomes

$$
f(T)=-g[T+f(T)]
$$

(where the subscript 0 on the temperature has been dropped). This equation allows the determination of $g$ given the measurement of $f$ (and vice versa). In Fig. 1 is shown corresponding $f$ and $g$ curves. It is clearly apparent that the shape and maximum point of the two curves differ. The general shape of the curves is one appropriate for pure materials (i.e., not containing grains of different composition and Curie temperature) where the adiabatic temperature change upon magnetization for a given $H$ has a single maximum at $T=T_{*}$, and no other local extrema. This maximum will be close to the Curie temperature $T_{C}$ but will, in general, not coincide with it; ${ }^{1}$ indeed, it will often depend on $H$.

Read from right to left Eq. (5) states that a material in field at a temperature $T+f(T)$ will, when demagnetized, cool to $T$. A magnetized material demagnetized from a starting temperature $T_{s}$ will cool to a unique temperature $T_{e}$ which obeys $T_{s}=T_{e}+f\left(T_{e}\right)$. The uniqueness implies that $T+f(T)$ is one-to-one considered as a function of temperature. Furthermore, the magnetized material may obviously be demagnetized from any starting temperature $T_{s}$ by connecting it to a heat bath at an appropriate temperature while in field; isolating it thermally; and then removing the field. This means that $T+f(T)$ must also map the entire temperature range $[0, \infty[$ onto itself. Taken together with the fact that it is one-to-one this implies that $T+f(T)$ is an invertible function. If $f(T)$ is continuous a necessary and sufficient condition for this to be the case is that $T+f(T)$ is monotonically increasing in the entire range $[0, \infty[$ (increasing, given that $f(T)$ approaches 0 for $T \rightarrow 0$ and $T \rightarrow \infty)$, i.e., that the derivative is greater than zero for all $T: d[T+f(T)] / d T>0$, or

$$
\frac{d\left[\Delta T_{\mathrm{mag}}(T, H)\right]}{d T}>-1 .
$$

This is the main result of the present paper; below we discuss how the inequality is modified for first-order materials. The same inequality will be obeyed by barocaloric materials (with $\Delta T$ being the change in temperature as the pressure is changed adiabatically from 0 to $p$ ) and electrocaloric materials (with $\Delta T$ being the change in temperature as the electric field is changed adiabatically from 0 to $E$ ), as long as the temperature change is a continuous function of $T$.

We note that assuming that $f$ and $g$ are differentiablewhich is a reasonable assumption for real materials, at most excepting a finite number of temperatures — we get, using the chain rule,

$$
f^{\prime}(T)=-\frac{1}{1+g^{\prime}[T+f(T)]^{-1}} .
$$

From this it is seen that if $f^{\prime}(T)$ approaches -1 at a given temperature $T_{1}$, the demagnetization curve $g$ becomes steeper and steeper, and when $f^{\prime}\left(T_{1}\right)$ reaches -1 the derivative of $g$ becomes infinite at the corresponding temperature $T_{1}+f\left(T_{1}\right)$, i.e., the curve becomes vertical at this point. This is shown on Fig. 1(b).

\section{FIRST-ORDER IRREVERSIBLE MATERIALS}

For magnetocaloric materials exhibiting a first-order phase transition, the magnetocaloric effect can be irreversible due to hysteretic losses. ${ }^{6}$ In such cases, the equality Eq. (2) is changed into an inequality,

$$
\Delta T_{\text {mag }}\left(T_{0}, H\right)>-\Delta T_{\text {demag }}\left[T_{0}+\Delta T_{\text {mag }}\left(T_{0}, H\right), H\right] .
$$

It is important to note that this irreversibility is limited to a temperature interval in the vicinity of the phase transition. ${ }^{6}$ Outside this temperature interval, the magnetocaloric effect is still reversible and the arguments of the previous section still apply, and, in particular, the constraint Eq. (6) applies.

Inside the irreversibility region, it is possible to use the general shape of the $T-S$ diagram for a first-order material to place limits on the variation in $\Delta T_{\text {mag }}{ }^{1}$ Consider such a material having a first-order phase transition from a lowtemperature phase to a high temperature at a temperature $T_{\mathrm{pt}, 1}$ in zero field. At a field $H$, the transition temperature will be $T_{\mathrm{pt}, 2}>T_{\mathrm{pt}, 1}$. Such a material will in the vicinity of the phase transition have an $T-S$ diagram as shown schematically in Fig. 2. For an ideal first-order transition, the entropy will be discontinous, i.e., the entropy curves will be vertical at $T_{\mathrm{pt}, 2}$ and $T_{\mathrm{pt}, 1}$, respectively. We now define a temperature by the following equation:

$$
S\left(T_{\mathrm{m}}, 0\right)=S\left(T_{\mathrm{pt}, 2}, H\right) .
$$

Above $T_{\mathrm{m}}, \Delta T_{\mathrm{mag}}$ will decrease linearly with $T$ until $T_{\mathrm{pt}, 2}$ is reached, as can be seen geometrically from the figure. This means that for $T_{\mathrm{m}}<T<T_{\mathrm{pt}, 2}$ we have the equality,

$$
\frac{d\left[\Delta T_{\mathrm{mag}}(T, H)\right]}{d T}=-1 .
$$




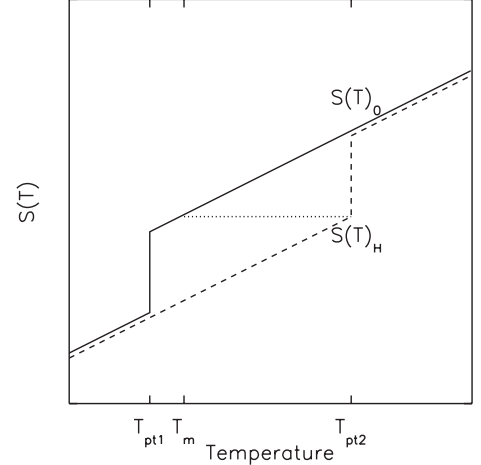

FIG. 2. Schematic $T-S$ diagram for a first-order material (after Ref. 1). Horizontal lines between the two curves with field 0 (full line) and $H$ (dashed line) correspond to the adiabatic temperature change $\Delta T_{\text {mag. }}$. The adiabatic temperature change has its maximum in the temperature interval between $T_{\mathrm{pt}, 1}$ and $T_{\mathrm{m}}$ (defined geometrically as shown). For $T_{\mathrm{m}}<T<T_{\mathrm{pt}, 2}$, the adiabatic temperature change decreases as $\Delta T_{\text {mag }}=T_{\mathrm{pt}, 2}-T$ due to the vertical entropy curve at $T_{\mathrm{pt}, 2}$. If the transition is not strictly first order, the entropy curve will have a finite, positive slope at $T_{\mathrm{pt}, 2}$ and the decrease in $\Delta T_{\text {mag }}$ will be slower.

In the interval $T_{\mathrm{pt}, 1}<T<T_{\mathrm{m}}$, the slope of the adiabatic temperature change is strictly greater than -1 . In this interval, the adiabatic temperature change attains its maximum value, which may be at more than one temperature. Indeed, direct measurements show plateau-like maximum adiabatic temperature changes. ${ }^{4}$

Thus, for first-order materials the strict inequality Eq. (6) is replaced by

$$
\frac{d\left[\Delta T_{\mathrm{mag}}(T, H)\right]}{d T} \geq-1,
$$

valid for all $T$.

\section{COMPARISON TO EXPERIMENTAL RESULTS}

The adiabatic temperature change may be measured experimentally in several different ways. Measuring the temperature change in a sample upon changing the field under adiabatic conditions $\Delta T_{\mathrm{mag}}=T(H)-T\left(H_{0}\right)$ is termed a direct method. The temperature change may also be calculated from entropy data using the relation $\Delta T_{\mathrm{mag}}=T^{*}-T_{0}$, where $S\left(T^{*}, H\right)=S\left(T_{0}, H_{0}\right)$. The entropy can be obtained either by integrating heat-capacity data or-using the Maxwell relation $\partial S / \partial H=\partial M / \partial T$ valid for materials with a second-order transition-through magnetization measurements. In the majority of the literature reporting the adiabatic temperature change in magnetocaloric materials, indirect methods are employed as standard equipment such as differential scanning calorimetry and magnetometry may be used for this. Direct measurements require more specialized, often custom built, equipment. Furthermore, even when direct measurements are used, usually only the magnetization results $\left(\Delta T_{\mathrm{mag}}\right)$ are reported. From magnetization results it is, however, straightforward to reconstruct the demagnetization curve from Eq. (2). In Fig. 3, we show a direct measurement

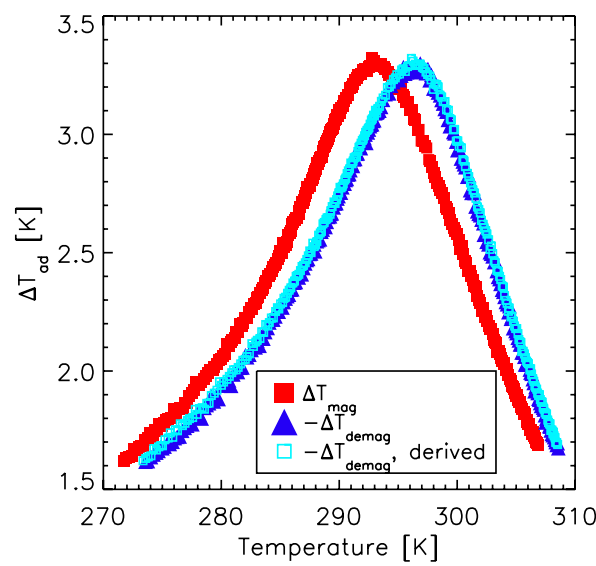

FIG. 3. (Color online) The adiabatic temperature change in gadolinium with a magnetic field change from 0 to $1.1 \mathrm{~T}$. Both $\Delta T_{\mathrm{mag}}$ and $-\Delta T_{\text {demag }}$ are shown (filled squares and triangles, respectively). The open squares show $-\Delta T_{\text {demag }}$ derived using Eq. (2) and the $\Delta T_{\text {mag }}$ data. The measurements were performed following a slightly modified procedure from Ref. 7 with the sample and magnet contained in a temperature-controlled environment.

of both $\Delta T_{\text {mag }}$ and $\Delta T_{\text {demag }}$ for a plate of $99.9 \%$ pure gadolinium (obtained from China Rare Metal Material Co.) together with the reconstructed demagnetization curve, showing the validity of this approach.

In second-order materials, good agreement is observed between $\Delta T_{\mathrm{mag}}$ results obtained by direct and indirect methods. ${ }^{8,9}$ However, for first-order materials severe discrepancies between the two methods are often observed. This is partly due to the slow kinetics of the structural part of the transition. ${ }^{10,11}$ Fast direct measurements may result in an underestimation of $\Delta T_{\mathrm{mag}}$. Also, the latent heat inherent to a first-order transition makes indirect methods relying on entropy results prone to erroneous results. ${ }^{12,13}$

First-order materials, in general, have a more abrupt change in $\Delta T_{\text {mag }}$ as a function of temperature, in agreement with the discussion above. When validating the derived constraint against experimental data we choose only to include directly measured temperature dependencies of $\Delta T_{\text {mag }}$ as any uncertainty in the results due to the kinetics of the transition will tend to underestimate the value of $\Delta T_{\text {mag. }}$.

A number of studies of first-order materials have shown direct measurements of $\Delta T_{\mathrm{mag}}$, where $\partial \Delta T_{\mathrm{mag}} / \partial T$ is close to the constraint of -1 , e.g., $\mathrm{MnAs},{ }^{14} \mathrm{La}\left(\mathrm{Fe}_{0.89} \mathrm{Si}_{0.11}\right)_{13},{ }^{15}$ $\mathrm{Mn}_{1} \mathrm{As}_{0.9} \mathrm{Sb}_{0.1},{ }^{16}$ and $\mathrm{Gd}_{5} \mathrm{Si}_{2} \mathrm{Ge}_{2} \cdot{ }^{17}$ It should be noted that the number of data points in the relevant temperature range just above $T_{*}$ where the slope of the $\Delta T_{\mathrm{mag}}$ is most negative in all of the cited studies is relatively limited, often consisting of only two to three measurements. As an example, in Fig. 4 the data from Ref. 16 is reproduced. $\Delta T_{\mathrm{mag}}$ data from field changes of $0-2$ and $0-5 \mathrm{~T}$ together with reconstructed values of $\Delta T_{\text {demag }}$ are shown. It is evident that the slopes of the magnetization curves are very close to -1 (corresponding to a vertical section of the demagnetization curves). However, within the experimental uncertainty it is not possible to determine whether the constraint indeed is violated. It would be interesting to resolve this temperature range in higher detail to allow a more stringent test of the constraint. 


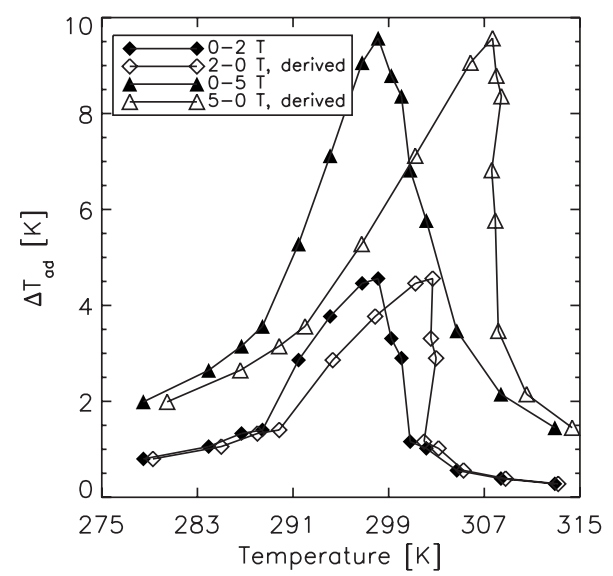

FIG. 4. The adiabatic temperature change in $\mathrm{Mn}_{1.00} \mathrm{As}_{0.9} \mathrm{Sb}_{0.1}$ when magnetizing from 0 to $2 \mathrm{~T}$ and from 0 to $5 \mathrm{~T}$. The curves denoted "derived" show the absolute value of the adiabatic temperature change when demagnetizing (changing the field from 2 to $0 \mathrm{~T}$ and from 5 to $0 \mathrm{~T}$, respectively) calculated from the $\Delta T_{\text {mag }}$ data set using Eq. (2). The data is reproduced from Ref. 16 and obtained through private communication with Dr. H. Wada.

\section{V. “DISCONTINUOUS” MATERIALS}

As shown above, a sufficient condition for the validity of the constraint, Eq. (6) (reversible materials) or Eq. (11) (irreversible materials), is that $f(T)=\Delta T_{\text {mag }}$ should be a continuous function of temperature. In this section, we show that a discontinuous, reversible $\Delta T_{\mathrm{mag}}$ can indeed violate the inequality. To do this we construct a model shape of a discontinuous $f(T)$ with $d f / d T<-1$ in a given interval. For simplicity we choose a constant slope $\alpha<-1$,

$$
f(T)= \begin{cases}\beta_{1}(T) & \text { for } T<T_{1} \\ \Delta T_{0}+\alpha\left(T-T_{1}\right) & \text { for } T_{1}<T<T_{2} . \\ \beta_{2}(T) & \text { for } T>T_{2}\end{cases}
$$

Here $T_{1}, T_{2}$, and $\Delta T_{0}$ are constants while $\beta_{1}$ and $\beta_{2}$ are arbitrary functions obeying $d \beta_{1} / d T>-1$ and $d \beta_{2} / d T>-1$, with the limiting values of $\beta_{1}\left(T_{1}\right)=\Delta T_{0}+(1+\alpha)\left(T_{2}-T_{1}\right)$ and $\beta_{2}\left(T_{2}\right)=\Delta T_{0}-\left(T_{2}-T_{1}\right)$. These values are chosen to make $T+f(T)$ invertible and thus ensure that Eq. (5) can be fulfilled for all temperatures. In Fig. 5, we show an example of such a discontinuous $f$ and the corresponding $g(T)=\Delta T_{\text {demag }}$.

It may be asked if such discontinuous materials actually exist. While we are aware of no direct reports in the literature of such magnetocaloric materials it is not completely inconceivable that they could exist. Consider, e.g., a material with competing structural and magnetic transitions. A lowtemperature magnetic state with a Curie temperature $T_{C 1}>T_{0}$ (or indeed a nonmagnetic state) is destabilized by a structural phase transition at $T=T_{0}$ in favor of a second magnetic state with a Curie temperature $T_{C 2} \leq T_{0}$. This second phase does not manifest itself at the low-temperature side of $T_{0}$ due to the structural phase transition. At $T=T_{1}$, the second phase is destroyed due to another structural instability in favor of a third phase with a Curie temperature $T_{C 3}$.

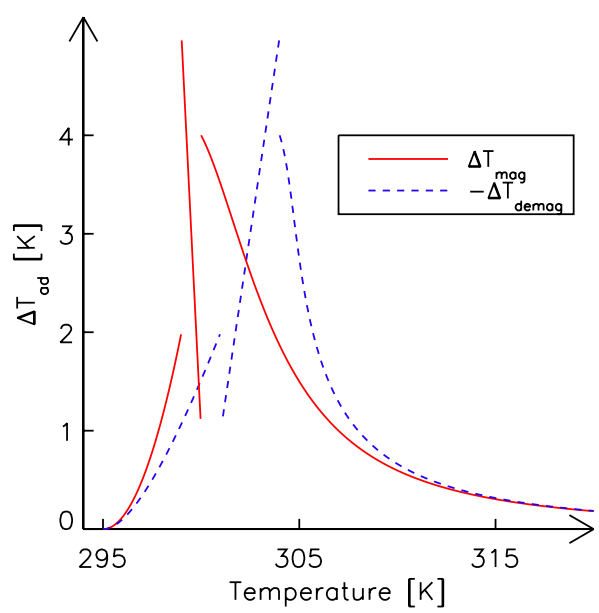

FIG. 5. (Color online) The adiabatic temperature change in a fictitious reversible magnetocaloric material with a discontinuous magnetocaloric effect. The full red line is the magnetization curve whereas the dashed blue line is the demagnetization curve. It is observed that such a material fulfills the reversibility criterion in Eq. (2) even though the slope of $\Delta T_{\text {mag }}$ is less than -1 over an entire temperature interval. Note that discontinuity is a necessity for the constraint in Eq. (6) to be invalid.

While such an interplay of phases may seem unlikely, the example at least shows that there are no obvious theoretical reasons forbidding a discontinuous variation in $\Delta T_{\mathrm{mag}}$ with temperature.

\section{DISCUSSION AND CONCLUSION}

A general constraint on the possible variation in the adiabatic temperature change at a fixed magnetic field change and as a function of temperature has been derived based on the basic thermodynamics of the magnetocaloric effect together with the assumption of continuity of the adiabatic temperature change as a function of temperature. These assumptions will apply to most real materials and as a result these materials will obey the constraint. This conclusion is in accordance with the literature of experimental data. However, better resolved data of the temperature region just above the maximum temperature change would be useful to be able to test the constraint in more detail.

The derived constraint will be of importance when optimizing graded regenerators for use in an active magnetic regenerative refrigerator device. Indeed it has been suggested that for an ideal performance the magnetocaloric effect of the regenerator should obey just this constraint. ${ }^{18}$ The results presented here show that for most materials this is a valid assumption.

\section{ACKNOWLEDGMENTS}

The authors thank the support of the Programme Commission on Energy and Environment (EnMi) (Contract No. 2104-06-0032) which is part of the Danish Council for Strategic Research. 
*Also at the Fuel Cells and Solid State Chemistry Division, Ris $\varnothing$ National Laboratory for Sustainable Energy, Technical University of Denmark; kaki@ risoe.dtu.dk

${ }^{1}$ V. K. Pecharsky, K. A. Gschneidner, Jr., A. O. Pecharsky, and A. M. Tishin, Phys. Rev. B 64, 144406 (2001).

${ }^{2}$ A. M. Tishin and Y. Spichkin, The Magnetocaloric Effect and its Applications (Institute of Physics, Bristol, 2003).

${ }^{3}$ P. J. von Ranke, N. A. de Oliviera, B. P. Alho, E. J. R. Plaza, V. S. R. de Sousa, L. Caron, and M. S. Reis, J. Phys.: Condens. Matter 21, 056004 (2009).

${ }^{4}$ R. Burriel, L. Tocado, E. Palacios, T. Tohei, and H. Wada, J. Magn. Magn. Mater. 290-291, 715 (2005).

${ }^{5}$ V. Franco, J. Blazquez, and A. Conde, Appl. Phys. Lett. 89, 222512 (2006).

${ }^{6}$ K. Morrison, J. D. Moore, K. G. Sandeman, A. D. Caplin, and L. F. Cohen, Phys. Rev. B 79, 134408 (2009).

${ }^{7}$ C. R. H. Bahl and K. K. Nielsen, J. Appl. Phys. 105, 013916 (2009).

${ }^{8}$ K. A. Gschneidner, Jr., V. K. Pecharsky, and A. O. Tsokol, Rep. Prog. Phys. 68, 1479 (2005).

${ }^{9}$ S. Y. Dan'kov, A. M. Tishin, V. K. Pecharsky, and K. A.
Gschneidner, Jr., Phys. Rev. B 57, 3478 (1998).

${ }^{10}$ A. Giguere, M. Foldeaki, B. Ravi Gopal, R. Chahine, T. K. Bose, A. Frydman, and J. A. Barclay, Phys. Rev. Lett. 83, 2262 (1999).

${ }^{11}$ A. Fujita, S. Fujieda, Y. Hasegawa, and K. Fukamichi, Phys. Rev. B 67, 104416 (2003).

${ }^{12}$ V. K. Pecharsky and K. A. Gschneidner, Jr., J. Appl. Phys. 86, 565 (1999).

${ }^{13}$ L. Tocado, E. Palacios, and R. Burriel, J. Appl. Phys. 105, 093918 (2009).

${ }^{14}$ L. Tocado, E. Palacios, and R. Burriel, J. Therm Anal. Calorim. 84, 213 (2006).

${ }^{15}$ A. Fujita and K. Fukamichi, J. Alloys Compd. 404-406, 554 (2005).

${ }^{16}$ H. Wada, T. Asano, M. Ilyn, and A. Tishin, J. Magn. Magn. Mater. 310, 2811 (2007).

${ }^{17}$ K. A. Gschneidner, V. K. Pecharsky, E. Bruck, H. G. M. Duijn, and E. M. Levin, Phys. Rev. Lett. 85, 4190 (2000).

${ }^{18}$ J. L. Hall, C. E. Reid, I. G. Spearing, and J. A. Barclay, Adv. Cryog. Eng. 41, 1653 (1996). 